\title{
Eligibility for shorter treatment of multidrug-resistant tuberculosis in the European Union
}

\author{
To the Editor:
}

In May 2016, the World Health Organization (WHO) updated its treatment guidelines for drug-resistant tuberculosis (TB) and included a recommendation for the use of the shorter multidrug-resistant (MDR) TB regimen [1]. The WHO update is based on information provided by observational studies coordinated by the International Union Against Tuberculosis and Lung Disease, Médecins sans Frontières and the Damien Foundation which showed that the shorter treatment regimen resulted in a higher likelihood of treatment success compared to the longer conventional treatment in the study settings [2-5]. The shorter MDR-TB regimen consists of a standardised treatment course lasting 9-12 months and includes kanamycin, moxifloxacin, prothionamide, clofazimine, pyrazinamide, isoniazid and ethambutol. It is recommended for patients with rifampicin-resistant (RR) or MDR-TB (cases with TB bacilli resistant to at least isoniazid and rifampicin) who have not been treated previously with second-line drugs and in whom resistance to fluoroquinolones and second-line injectable agents has been excluded or is considered highly unlikely. It is not recommended for pregnant women and patients with extrapulmonary TB.

In the European Union (EU)/European Economic Area (EEA), 1500 MDR-TB cases are reported annually [6] and information on all TB cases is reported to the European Surveillance System (TESSy) hosted by the European Centre for Disease Prevention and Control. We used this case-based database to assess the potential for the use of the shorter treatment regimen within the EU/EEA. The WHO defined the following criteria for eligibility for the shorter MDR-TB regimen: pulmonary TB; no infection with strains known or strongly suspected of being resistant to one or more drugs in the shorter MDR-TB treatment regimen except for isoniazid; no exposure for $\geqslant 1$ months to second-line medicines included in the shorter MDR-TB regimen; no intolerance to medicines in the shorter MDR-TB regimen or risk of toxicity; and no pregnancy TB [1]. TESSy holds information on the site of disease (pulmonary and extrapulmonary) and previous treatment, and drug susceptibility testing (DST) results are collected for isoniazid, rifampicin, ethambutol, streptomycin, amikacin, kanamycin, capreomycin, ciprofloxacin, ofloxacin, levofloxacin, gatifloxacin and moxifloxacin [6], but not for prothionamide, clofazimine and pyrazinamide. DST results are not collected for pyrazinamide, because of concerns regarding test validity $[7,8]$, while DST data for clofazimine and prothionamide are not collected following WHO recommendations [9]. Information on exposure to specific second-line drugs included in the shorter regimen is not collected, therefore we only included cases that were not previously treated, i.e. new cases. Information on tolerance or expected toxicity is also not available. The TESSy TB dataset does not contain information on pregnancy.

We assessed the number and proportion of RR-/MDR-TB cases in the EU/EEA that would be eligible for the shorter treatment regimen among all RR-/MDR-TB cases reported between 2010 and 2014. Data from 26 EU/EEA countries providing case-based DST results were included: Austria, Belgium, Bulgaria, Croatia, Cyprus, Czech Republic, Denmark, Estonia, Finland, Germany, Greece, Hungary, Iceland, Ireland, Latvia, Lithuania, Luxembourg, Malta, the Netherlands, Norway, Poland, Portugal, Romania, Slovakia, Sweden, and United Kingdom. Since there is a high level of cross-resistance between kanamycin and amikacin, we considered a case as kanamycin-resistant if resistance was reported for one of the two drugs [10]. Due to cross-resistance between ofloxacin and moxifloxacin, we considered ofloxacin-resistant cases as moxifloxacin-resistant [11]. We used these assumptions because the testing coverage for kanamycin and moxifloxacin was lower compared to the coverage of amikacin and ofloxacin.

@ERSpublications

In the European Union, $11 \%$ of all MDR-TB cases are eligible for the shorter MDR-TB regimen http://ow.ly/kukg306AcFl

Cite this article as: van der Werf MJ, Hollo V, Ködmön C, et al. Eligibility for shorter treatment of multidrug-resistant tuberculosis in the European Union. Eur Respir J 2017; 49: 1601992 [https://doi.org/ 10.1183/13993003.01992-2016]. 
Between 2010 and 2014, 7550 RR-/MDR-TB episodes were notified in the EU/EEA. Of these, 3103 (41.1\%) were new, and among these new cases, 2839 (91.5\%) were new pulmonary RR-/MDR-TB cases (figure 1 and table 1). 1774 (62.5\%) new pulmonary RR-/MDR-TB cases had results from second-line DST for kanamycin/amikacin, moxifloxacin/ofloxacin and ethambutol, of which 1250 (70.5\%) showed resistance to at least one of the drugs excluding them from the shorter regimen.

Thus, following the criteria specified by the WHO, 524 RR-/MDR-TB cases diagnosed in 2010-2014 would be eligible for the shorter regimen based on their treatment history, site of disease and DST results. Since second-line DST results were only available for 1774 (62.5\%) of all new pulmonary RR-/MDR-TB cases, the actual number of eligible cases might be higher. Assuming that those without DST results would have a similar pattern of resistance as those with results, we might expect 839 RR-/MDR-TB cases eligible for the new shorter treatment, or $11.1 \%$ of all notified RR-/MDR-TB cases. This is probably a conservative estimate, since MDR-TB cases without second-line DST results may not have been tested because clinicians perceived them to have a low risk for second-line drug resistance, and thus the assumption that MDR-TB cases without second-line DST results cases have a similar pattern of resistance to those with results would underestimate the proportion eligible for the shorter MDR-TB regimen. In addition, MDR-TB cases that were previously treated and thus excluded from our analysis may be eligible for the shorter MDR-TB regimen, which further underestimates the proportion eligible for the shorter MDR-TB regimen. However, it is also possible that second-line DST results were not available because of limited access to these services, in which case our assumption of similar resistance patterns would be valid.

The shorter MDR-TB regimen is an important step forward, making MDR-TB treatment easier and less expensive [12]. However, in the EU/EEA, our conservative estimate is that only one in 10 RR-/MDR-TB cases are eligible for the shorter regimen. Similar low proportions have been reported in other studies [13].

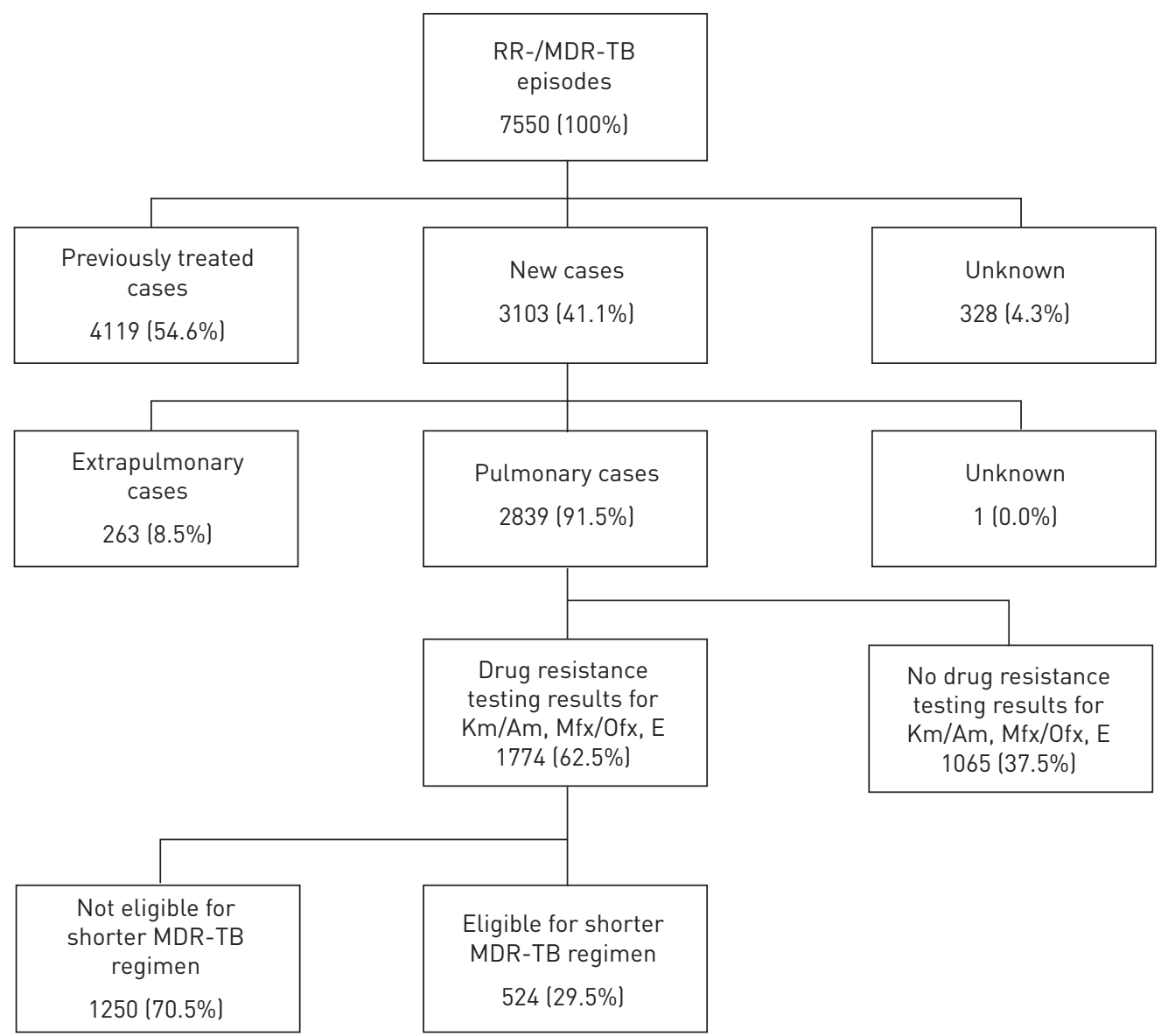

FIGURE 1 Rifampicin-resistant (RR-) and multidrug-resistant tuberculosis (MDR-TB) cases eligible for the shorter MDR-TB regimen in the European Union and the European Economic Area countries (Austria, Belgium, Bulgaria, Croatia, Cyprus, Czech Republic, Denmark, Estonia, Finland, Germany, Greece, Hungary, Iceland, Ireland, Latvia, Lithuania, Luxembourg, Malta, the Netherlands, Norway, Poland, Portugal, Romania, Slovakia, Sweden and the United Kingdom), 2010-2014. Km: Kanamycin; Am: Amikacin; Mfx: moxifloxacin; Ofx: ofloxacin; E: ethambutol. 
TABLE 1 Proportional prevalence of anti-tuberculosis (TB) drug-resistance by treatment history in rifampicin-resistant (RR)-/ multidrug-resistant (MDR)-TB cases in the European Union and European Economic Area countries 2010-2014.

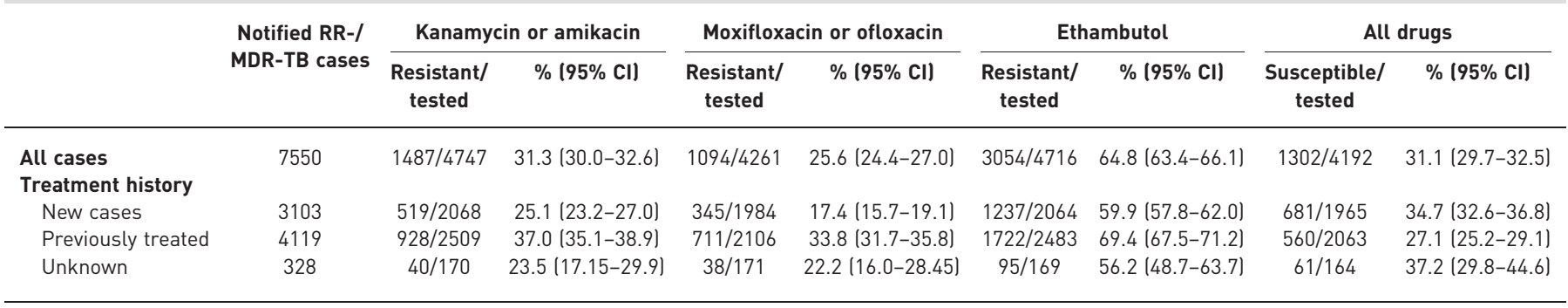

Data are presented as n, unless otherwise stated. Data from Austria, Belgium, Bulgaria, Croatia, Cyprus, Czech Republic, Denmark, Estonia, Finland, Germany, Greece, Hungary, Iceland, Ireland, Latvia, Lithuania, Luxembourg, Malta, the Netherlands, Norway, Poland, Portugal, Romania, Slovakia, Sweden and the United Kingdom.

In addition, given the low treatment success rate of $40.7 \%$ of the 2012 MDR-TB cohort [6], there is a need for better treatment for those not eligible for the shorter MDR-TB regimen. Further evaluation of the shorter regimen is ongoing in a randomised controlled study as part of the STREAM (Evaluation of a Standardised Treatment Regimen of Anti-tuberculosis Drugs for Patients with MDR-TB) trials [14]. This may provide evidence on the effectiveness of the regimen for those currently not eligible. In addition, other regimens are tested for treatment of MDR-TB, including an all-oral regimen [15], which would further simplify MDR-TB treatment.

In conclusion, the shorter MDR-TB regimen is an important step forward in improving treatment of MDR-TB and will benefit MDR-TB cases in the EU/EEA. However, further steps are badly needed.

Marieke J. van der Werf ${ }^{1}$, Vahur Hollo ${ }^{1}$, Csaba Ködmön ${ }^{1}$, Masoud Dara ${ }^{2}$ and Mike Catchpole ${ }^{1}$

${ }^{1}$ European Centre for Disease Prevention and Control, Stockholm, Sweden. ${ }^{2}$ World Health Organization Regional Office for Europe, Copenhagen, Denmark.

Correspondence: Marieke J. van der Werf, Tombtebodavägen 11a, 117165 Solna, Sweden.

E-mail: marieke.vanderwerf@ecdc.europa.eu

Received: Oct 112016 | Accepted after revision: Nov 152016

Conflict of interest: None declared.

Acknowledgements: The authors acknowledge the contribution of the nominated tuberculosis surveillance experts of the EU/EEA member states for providing the data to TESSy: Alexander Indra (Austrian Agency for Health and Food Safety, Vienna, Austria), Maryse Wanlin (Respiratory Diseases Fund, Brussels, Belgium), Tonka Varleva (Ministry of Health, Sofia, Bulgaria), Aleksandar Simunovic (Croatian Institute of Public Health, Zagreb, Croatia), Maria Koliou (Directorate of Medical and Public Health Services, Nicosia, Cyprus), Jiri Wallenfels (University Hospital Bulovka, Prague, Czech Republic), Peter Henrik Andersen (Statens Serum Institut, Copenhagen, Denmark), Piret Viiklepp (National Institute for Health Development, Tallinn, Estonia), Hanna Soini (National Institute for Health and Welfare, Turku, Finland), Walter Haas (Robert Koch Institute, Berlin, Germany), Ourania Kalkouni (Hellenic Centre for Disease Control and Prevention, Athens, Greece), Gábor Kovács (Koranyi National Institute of Tuberculosis and Pulmonology, Budapest, Hungary), Thorsteinn Blondal (Primary Health Care Centre Capital Area, Reykjavik, Iceland), Joan O'Donnell (Health Protection Surveillance Centre, Dublin, Ireland), Dace Mihalovska (Centre for Disease Prevention and Control, Riga, Latvia), Edita Davidavičienè (National Tuberculosis and Infectious Diseases University Hospital, Vilnius, Lithuania), Irene Demuth (Health Directorate, Luxembourg, Luxembourg), Analita Pace Asciak (Superintendence of Public Health, Valletta, Malta), Erika Slump (National Institute for Public Health and the Environment, Bilthoven, the Netherlands), Trude Margrete Arnesen (National Institute of Public Health, Oslo, Norway), Maria Korzeniewska-Koseła (National Tuberculosis and Lung Disease Institute, Warsaw, Poland), Antonio Diniz (Directorate General of Health, Lisbon, Portugal), Domnica Ioana Chiotan (Institute of Pneumology "Marius Nasta", Bucharest, Romania), Jerker Jonsson (Public Health Agency of Sweden, Stockholm, Sweden), Petra Svetina (University Clinic of Respiratory and Allergic Diseases Golnik, Golnik, Slovenia), Ivan Solovic (National Institute for Tuberculosis, Lung Diseases and Thoracic Surgery, Vyšné Hágy, Slovakia) and Maeve Lalor (Public Health England, London, UK)

\section{References}

1 World Health Organization (WHO). WHO Treatment Guidelines for Drug-resistant Tuberculosis - 2016 Update. Geneva, World Health Organization, 2016.

2 Aung KJ, Van Deun A, Declercq E, et al. Successful '9-month Bangladesh regimen' for multidrug-resistant tuberculosis among over 500 consecutive patients. Int J Tuberc Lung Dis 2014; 18: 1180-1187.

3 Kuaban C, Noeske J, Rieder HL, et al. High effectiveness of a 12-month regimen for MDR-TB patients in Cameroon. Int J Tuberc Lung Dis 2015; 19: 517-524.

4 Piubello A, Harouna SH, Souleymane MB, et al. High cure rate with standardised short-course multidrug-resistant tuberculosis treatment in Niger: no relapses. Int J Tuberc Lung Dis 2014; 18: 1188-1194. 
5 Van Deun A, Maug AK, Salim MA, et al. Short, highly effective, and inexpensive standardized treatment of multidrug-resistant tuberculosis. Am J Respir Crit Care Med 2010; 182: 684-692.

6 European Centre for Disease Prevention and Control, World Health Organization Regional Office for Europe. Tuberculosis Surveillance and Monitoring in Europe, 2016. Stockholm, European Centre for Disease Prevention and Control, 2016.

7 Hewlett D Jr, Horn DL, Alfalla C. Drug-resistant tuberculosis: inconsistent results of pyrazinamide susceptibility testing. JAMA 1995; 273: 916-917.

8 Heifets L. Susceptibility testing of Mycobacterium tuberculosis to pyrazinamide. J Med Microbiol 2002; 51: 11-12.

9 World Health Organization. Policy Guidance on Drug-susceptibility Testing (DST) of Second-line Antituberculosis Drugs. Geneva, World Health Organization, 2008.

10 Jugheli L, Bzekalava N, de Rijk P, et al. High level of cross-resistance between kanamycin, amikacin, and capreomycin among Mycobacterium tuberculosis isolates from Georgia and a close relation with mutations in the rrs gene. Antimicrob Agents Chemother 2009; 53: 5064-5068.

11 Willby M, Sikes RD, Malik S, et al. Correlation between GyrA substitutions and ofloxacin, levofloxacin, and moxifloxacin cross-resistance in Mycobacterium tuberculosis. Antimicrob Agents Chemother 2015; 59: 5427-5434.

12 Sotgiu G, Tiberi S, D'Ambrosio L, et al. WHO recommendations on shorter treatment of multidrug-resistant tuberculosis. Lancet 2016; 387: 2486-2487.

13 Sotgiu G, Tiberi S, D’Ambrosio L, et al. Faster for less: the new "shorter" regimen for multidrug-resistant tuberculosis. Eur Respir J 2016; 48: 1503-1507.

14 Moodley R, Godec TR, STREAM Trial Team. Short-course treatment for multidrug-resistant tuberculosis: the STREAM trials. Eur Respir Rev 2016; 25: 29-35.

15 Wallis RS, Maeurer M, Mwaba P, et al. Tuberculosis - advances in development of new drugs, treatment regimens, host-directed therapies, and biomarkers. Lancet Infect Dis 2016; 16: e34-e46.

Copyright @ERS 2017

This ERJ Open article is open access and distributed under the terms of the Creative Commons Attribution Non-Commercial Licence 4.0. 